\title{
Advance information and movement sequencing in Gilles de la Tourette's syndrome
}

\author{
Nellie Georgiou, John L Bradshaw, Jim G Phillips, Judy A Bradshaw, Edmond Chiu
}

\begin{abstract}
Tourette's syndrome is a chronic neurological disorder manifested by involuntary motor tics and vocalisations. Because the basal ganglia have been implicated in the pathology underlying Tourette's syndrome, the present two procedures, both involving sequential movements, sought to determine the extent to which patients with Tourette's syndrome were reliant on, and could utilise different levels of advance information. Patients with Tourette's syndrome were found to be more reliant than controls on external visual cues to execute rather than to initiate a motor programme. When there was a high level of reduction in advance informationthat is, a visual pathway to be followed was extinguished well in advance of each successive movement-executions progressively slowed as the sequence was traversed. Similarly, if no advance information was provided before each move, movement execution was slower than that of controls. The movement initiation times of patients with Tourette's syndrome were, however, similar to those of controls, as were their movement execution times when advance visual information was available. It seems that patients with Tourette's syndrome, like parkinsonian patients who are known to have a basal ganglia disorder, require external sensory cues to sequence a motor programme effectively. The present study found evidence consistent with the hypothesis that patients with Tourette's syndrome, like patients with Parkinson's disease, may be dysfunctional in internal switching mechanisms. Alternatively, with limited visual guidance, patients with Tourette's syndrome, regardless of medication or depression state, may require more time to plan and programme each next submovement, and under such conditions may require external visual cues to direct attention effectively to given targets. Although the underlying pathogenesis is still speculative, it is concluded that there is much to support the notion that Tourette's syndrome may stem from abnormalities of the major pathways between the basal ganglia and the frontal lobes.
\end{abstract}

(F Neurol Neurosurg Psychiatry 1995;58:184-191)
Keywords: Tourette's syndrome; basal ganglia; frontal lobes; advance information.

Gilles de la Tourette's syndrome is a movement disorder manifesting as involuntary motor and sometimes vocal tics. The tics themselves are characterised by sudden muscle jerks occurring at irregular intervals. Simple tics occur most often in the facial musculature, although they can also include, for example, a shrug of a shoulder or a jerk of an arm. More complex movements can also appear, such as touching, wiping, or hitting. ${ }^{1}$ In addition, patients with Tourette's syndrome often have other associated disorders. For example about $60 \%$ may have attention deficit hyperactivity disorder. ${ }^{2}$ Other symptoms include sleep disorders, palilalia (compulsive repetition of one's own sounds or words), echolalia (repeating another's words or sounds), echopraxia (repeating one's movements), copropraxia (performing socially unacceptable actions), and coprolalia (uttering socially inappropriate words). In about $50 \%$ of cases, obsessive compulsive behaviour is noted, including intrusive sexual thoughts and actions. ${ }^{3}$

Recent evidence suggests that Tourette's syndrome is inherited by autosomal dominant transmission, and that the syndrome may be the result of dopaminergic dysfunction arising from disturbances in the midbrain, ${ }^{34}$ although there may also be disturbances of other neurotransmitters such as serotonin, $\gamma$-aminobutyric acid, noradrenaline, and acetylcholine. Dopaminergic fibres project from the substantia nigra to the striatum, and from the ventral tegmental area to the frontal lobes. ${ }^{5}$ Tourette's syndrome may stem from abnormalities of these major fibre pathways, from lesions in other brain regions that have primary interconnections with the basal ganglia, and from alterations within the basal ganglia themselves. ${ }^{6}$ Hallett $^{7}$ suggests that the disorder may be associated with an overactivity of the pathway that links the putamen and the internal division of the globus pallidus. The nature of the overactivity is probably different between patients with Tourette's syndrome and those with other movement disorders (for example, Huntington's disease and the dyskinesiae) as the tics associated with Tourette's syndrome are distinct.

In support of the view of involvement of the basal ganglia in the aetiology of Tourette's syndrome, various researchers ${ }^{89}$ have reported that the lenticular region (putamen and 
globus pallidus) of the basal ganglia in the left hemisphere of patients with Tourette's syndrome is reduced in volume compared with the control group. In association with this, there seems to be a left side predominance in Tourette's syndrome (reduced basal ganglia volume) relative to the right side predominance in control subjects. Despite the fact that in both of these studies there were numerous methodological differences (for example, age of patients, technical specifications of MRI, and systems of measurement) these results are extraordinary given the similarities of their findings. Both studies conclude that the basal ganglia are specifically involved in the pathogenesis of Tourette's syndrome.

We have previously examined two other disorders of the basal ganglia-namely, Parkinson's disease ${ }^{1011}$ and Huntington's disease, ${ }^{12}$ finding deficits in the accurate running off or sequencing of complex motor programmes. These deficits are most evident in tasks requiring some degree of automaticity, and in particular when sequential movements have to be controlled in the absence of external visual cues, ${ }^{10}$ or when there are low levels of advance information. ${ }^{12} 13$

In view of the suggested involvement of the basal ganglia in Tourette's syndrome, a disorder which hitherto has received little experimental attention, we sought to extend our techniques to that syndrome. We have developed a serial choice button pressing procedure that can independently assess two indices of response time-button "down time" (DT) and "movement time" (MT). The first, DT, which measures how long each button is held down before initiating a move to the next button in the sequence, is thought partly to reflect preparation time. The second, MT, is the time "in flight" between the release of one button and the depression of the next, and reflects execution time.

Two tasks that we have previously developed $^{1012}$ were adopted in the present study with a group of patients with Tourette's syndrome. The first task examined the patients' reliance on advance information, by presenting a series of illuminated buttons at a number of choice points along a response board. This procedure enables a sequential pathway to be designated, and with each successive button press visual information (advance information about the pathway ahead) can be systematically reduced by extinguishing lights, to various extents, in advance of each move. Patients were required to perform a sequence of 10 visually cued button press responses along a visually illuminated pathway down a response board. This task, which involves sequential forced choice responding, also permits measurement of any progressive slowing as the sequence is traversed as a function of how advance information is reduced. In particular, we sought to determine the extent to which patients with Tourette's syndrome rely on external visual cues to programme and sequence movements. As Singer and Walkup ${ }^{6}$ have suggested that there may be deficits in the "executive function domain," patients with Tourette's syndrome may show deficits in their ability to sequence together complex behaviours and to plan strategies ahead of time.

Based on these previous suggestions, it was therefore predicted that patients with Tourette's syndrome may show difficulty in initiating and executing sequential movements with high levels of reduction in advance information-that is, when they have to plan strategies of execution in advance of their own movement. We also predicted, on the basis of our findings, ${ }^{10}$ that patients with Tourette's syndrome would progressively slow in response execution as they moved down the board under a high level of reduction in advance information.

Whereas the first technique assessed patients' reliance on the continued availability of advance information, differentially deleting it ahead of each move, the second task examined the utilisation of advance information by adding it at different points in each response cycle. Thus motor programming was varied using three levels of advance information for each upcoming movement, again adapting our technique. ${ }^{12}$ Contrary to the first procedure, however, where target lights extinguished one at a time in advance, patients this time, while again being required to conduct movements between a series of 10 sequential buttons, had the target buttons illuminated one at a time as they progressed along the board. Simple motor programming was therefore varied by illuminating each target button, to various extents, increasingly further ahead of the current movement. Based on previous findings with both Parkinson's disease ${ }^{10}$ and Huntington's disease, ${ }^{12}$ it was predicted that patients with Tourette's syndrome might experience difficulty in performing movements with both low and high levels of advance information - that is, where no visual cue was presented in advance of the patient's own moves, or when it was presented two moves ahead, and both the next and the next but one move had to be simultaneously borne in mind progressively down the board.

\section{Methods}

SUBJECTS

Twelve patients with Tourette's syndrome, all volunteers recruited from the Victorian Tourette Syndrome Association, and 12 age matched controls with no history of neurological disorder participated. Patients with Tourette's syndrome met DSM-III-R ${ }^{14}$ diagnostic criteria for Tourette's syndrome with chronic motor and vocal tics, onset before the age of 21 years, and duration of tics of more than one year. One patient with evidence of mental retardation was excluded. There were 11 men and one woman in each group, all of whom were right handed with a mean age of 29 years for each group. Duration of Tourette's syndrome ranged from 1.5-13 years with a mean duration of 6.3 years. The table shows the clinical data for the Tourette's 
Clinical data for patients with Tourette's syndrome

\begin{tabular}{llllll}
\hline $\begin{array}{l}\text { Patient } \\
\text { No }\end{array}$ & Age & Sex & $\begin{array}{l}\text { Duration of } \\
\text { Tourette's syndrome }\end{array}$ & $\begin{array}{l}\text { Depression } \\
\text { score }\end{array}$ & $\begin{array}{l}\text { Medication for } \\
\text { Tourette's syndrome }\end{array}$ \\
\hline 1 & 57 & M & 3 & 10 & Thioridazine \\
2 & 25 & M & 5 & 19 & Pimozide \\
3 & 24 & M & 10 & 1 & Fluoxetine \\
4 & 26 & M & $1 \cdot 5$ & 13 & - \\
5 & 54 & M & 5 & 14 & - \\
6 & 27 & M & 2 & 6 & Fluoxetine \\
7 & 37 & F & 3 & 12 & Clomipramine \\
8 & 19 & M & 12 & 2 & - \\
9 & 18 & M & 2 & 1 & Pimozide \\
10 & 24 & M & 13 & 7 & Haloperidol \\
11 & 18 & M & 9 & 25 & M \\
12 & 21 & M & 9 & 14 & .
\end{tabular}

Patients $2,5,6,7,9$, and 10 were given no medication, and patients $1,3,7,9,10$, and 11 were classified as not depressed. ${ }^{16}$
$20 \mathrm{~mm}$ from the board and was $13 \mathrm{~mm}$ in diameter (fig 1).

Adjacent buttons were $30 \mathrm{~mm}$ apart, as were the rows themselves. In addition, two single buttons (S1, S2) were sequentially pressed to initiate the task, and a further single button $(F)$ at the other end of the board was pressed to complete a trial. Each button was illuminated by a light emitting diode set into its base. A computer recorded the time that each button was held down (down time, DT) and the time between the release of one button and the depression of the next (movement time, MT). It also determined the button sequence to be followed (the illuminated path) at the beginning of each trial.

\section{PROCEDURE}

For both tasks, the board was positioned across the subjects' midline, with the two start buttons ( $\mathrm{S} 1$ and $\mathrm{S} 2$ ) closest to the right side of the body. One of each of the two buttons at each of the 10 choice points was to be pressed with the index finger of the right hand as quickly and as accurately as possible. Each trial was completed after the 10th button in the series had been pressed, and the subject had reached button $F$. If a button was incorrectly pressed or was pressed twice the trial was terminated and the occurrence was recorded as an error. Data from such trials were not included in the analyses and the trial was repeated. Moreover, any movements that were disrupted by tics were excluded from the analyses. Finally, there were eight different equidistant pathways for both tasks, in which the number of linear and diagonal movements between successive button pairs were balanced, so that overall, the total distance in any one movement sequence was invariant. The different conditions were counterbalanced across subjects, with 16 experimental and six practice trials for each of the three cue conditions.

\section{RELIANCE ON ADVANCE INFORMATION}

In this task, a pathway of illuminated lights was presented down the board, with two way choice points at each of the 10 sequential pairs of buttons. Advance information (external visual cues) was systematically reduced (the illuminated buttons extinguished in advance of the next movement) in one of three ways as the subject progressed along the board: (a) with no reduction in advance information, the next illuminated button was extinguished as the current button was released (no button extinguished in advance); (b) with moderate reduction, the next button was extinguished as the current button was depressed (one button extinguished in advance); and (c) with a high level of reduction in advance information, in addition to the next button remaining extinguished, the next but one button also extinguished as the current button was released.

UTILISATION OF ADVANCE INFORMATION

In this task, instead of being presented with a pathway of illuminated lights down the board, 
Figure 2 Mean down time (DT) and movement time (MT) against levels of reduction in advance information for both Tourette's syndrome (TS) and control subjects (Con); bars are SEM.

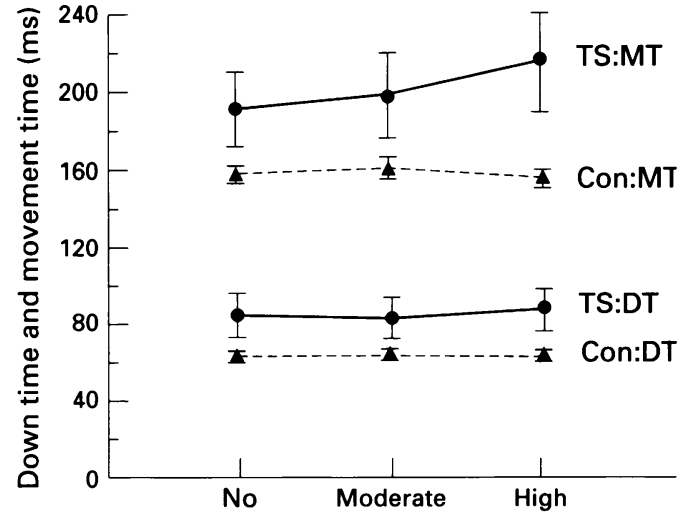

Levels of reduction in advance information

subjects were given no prior knowledge about the designated pathway until they started to press the two start buttons to initiate the task. With each successive button press, the next target button was illuminated in one of three ways: (a) with a low level of advance information, the next button to be depressed was illuminated as the current button was released, and subjects had to prepare their responses "on-line"; (b) with a moderate level of advance information, the next button to be depressed was illuminated as the current button was depressed; and $(c)$ with a high level of advance information, the next but one button was illuminated as the current button was released.

Although each button remained illuminated until the subject had depressed the next button in the series, it would nevertheless extinguish if not pressed within $1000 \mathrm{~ms}$.

\section{Results}

RELIANCE ON ADVANCE INFORMATION

Down time and sequencing effects

Figure 2 shows DT plotted as a function of reduction in advance information for each group. The data were submitted to a mixed two way ANOVA (group, reduction in advance information), which showed no significant main effects and no significant interaction $(F(2,44)=0.49, \mathrm{p}>0.62)$. Moreover, a trend analysis showed no evidence of progressive sequential slowing in either group or for any condition, despite the significant overall main effect of sequence $(F(9,360)=21 \cdot 92$, $\mathrm{p}<0.01$, fig 3).

In the DT analysis, therefore, patients with Tourette's syndrome were able to initiate and sequence movements as adequately as controls across the three cue conditions without any evidence of abnormal progressive slowing across the board.

Movement time and sequencing effects

Figure 2 also provides MT data. Although the main effect for group just failed to reach significance $(F(1,22)=3 \cdot 78, \mathrm{p}>0 \cdot 065)$, there was a significant main effect of reduction in advance information $(F(1,22)=3.53, \mathrm{p}<$ 0.05 ) and a highly significant interaction of group by reduction in advance information $(F(2,44)=6.03, \mathrm{p}<0.01)$. Post hoc one way ANOVAs and Tukey tests showed that whereas in controls there were no differences between any of the three levels of reduction in advance information, patients with Tourette's syndrome showed significant $(p<0.05)$ differences between high $(215 \mathrm{~ms})$ and no reduction $(191 \mathrm{~ms})$, thus indicating that continued provision of advance information assisted in their effective sequencing of movements. To interpret the sequencing effects further, a trend analysis was carried out. This showed that there was a significant main effect of sequence $(F(9,360)=5.68, \mathrm{p}<0.001)$, which had a significant $(\mathrm{p}<0.01)$ linear component and an almost significant group by sequence interaction $(F(9,720)=1.88, \mathrm{p}<0.056$, fig 3). Patients with Tourette's syndrome displayed considerable difficulty in progressively executing movements with the most reduced level of advance information. As a consequence, movements tended to progressively slow with each successive element in the response sequence.

Finally, the median errors for the three conditions (no, moderate, high) were 0,2 , and 5 for patients with Tourette's syndrome, and 0,2 , and 3 for controls respectively. Mann-Whitney $U$ tests showed that these group differences were not significant.

\section{Medication}

The question of whether medication may have affected Tourette's syndrome perfor-
Figure 3 Mean down time and movement time against each of the 10 sequential buttons for both Tourette's syndrome (TS) and control subjects (Con).
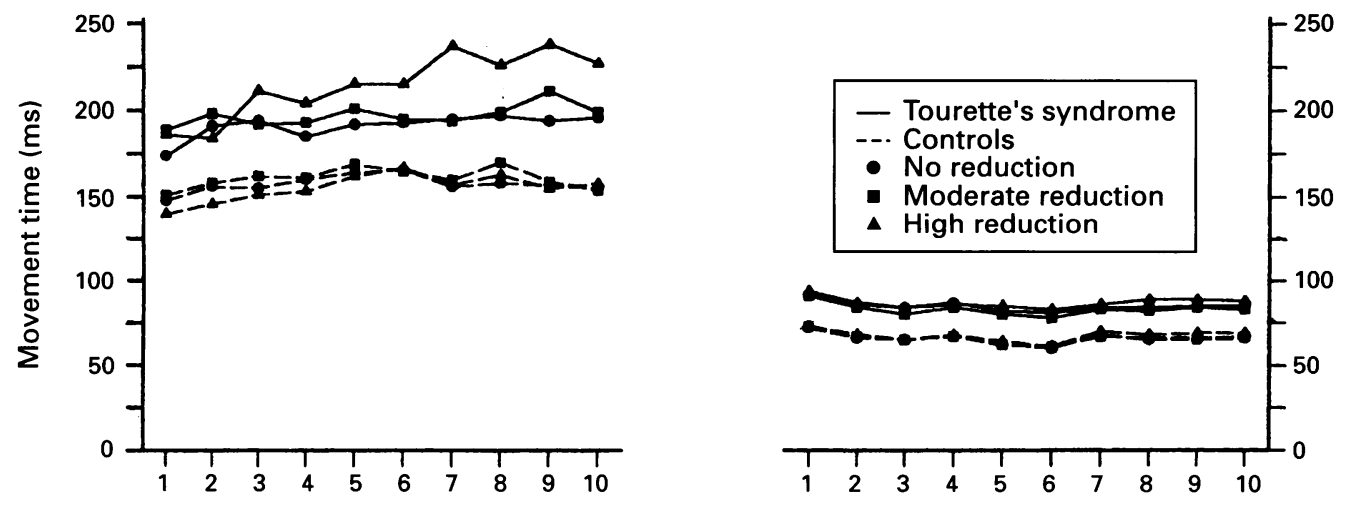
Figure 4 Mean down time (DT) and movement time (MT) against levels of advance information for both Tourette's syndrome (TS) and control subjects (Con); bars are SEM.

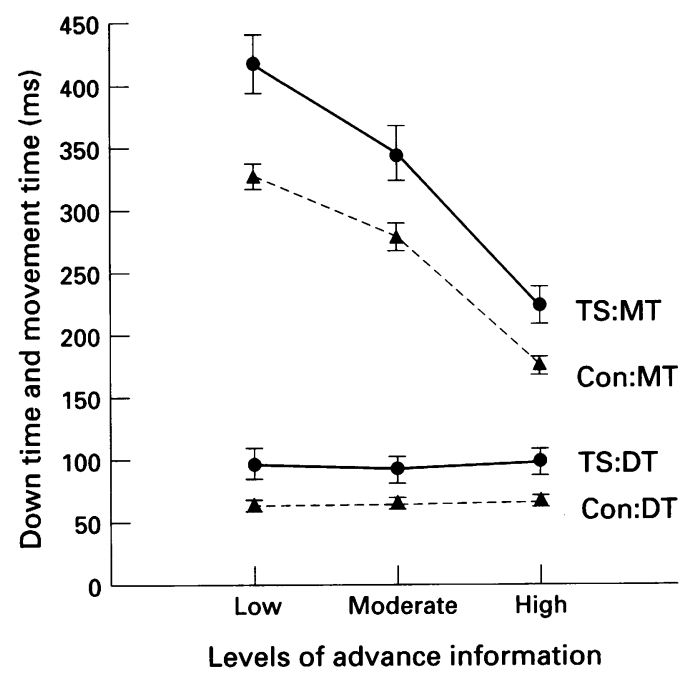

mance was considered. As six of the patients with Tourette's syndrome were not given medication, the group was divided into medicated and not medicated, and an ANOVA was conducted only on the MT data, as no significant interaction had been found in the DT data between controls and patients with Tourette's syndrome. Although there was no significant main effect of medication (medicated $v$ not medicated; $F(1,10)=3 \cdot 67, \mathrm{p}>$ 0.09 ), there was a significant main effect of reduction in advance information $(F(1,10)=$ $5.62, \mathrm{p}<0.05)$; most importantly, there was no significant interaction of medication by reduction in advance information $(F(2,20)=$ $1 \cdot 43, \mathrm{p}>0 \cdot 26)$. In absolute terms, the magnitude of the experimental effect (the difference between the first and third condition) was larger for the medicated group than for the not medicated group ( $29 \mathrm{~ms}$ and $12 \mathrm{~ms}$ ). Overall, patients with Tourette's syndrome medicated or not, experienced similar difficulty when obliged to rely upon limited advance information.

\section{Depression}

Because only six of the 12 patients with Tourette's syndrome could be classified as depressed (table), the group was subdivided into depressed and not depressed, and an ANOVA was conducted, again only on the MT data, to determine whether performance across each of the three conditions was differentially affected by depression. Although there was no significant main effect of depression (depressed $v$ not depressed; $F(1,10)=$ $1 \cdot 03, p>0.33)$, there was of course a significant main effect of reduction in advance information $(F(1,10)=4.67, \mathrm{p}<0.05)$; most importantly, there was no significant interaction of depression by reduction in advance information $(F(2,20)=0.05, \mathrm{p}>0.96)$. The magnitude of the experimental effect resides in the difference between the first and third condition, which was therefore similar for the depressed $(26 \mathrm{~ms})$ and the not depressed (22 ms) group. Patients with Tourette's syndrome, therefore, whether depressed or not, experienced similar difficulties when obliged to rely on reduced levels of advance information.

UTILISATION OF ADVANCE INFORMATION

Down time and sequencing effects

Figure 4 shows DT plotted as a function of advance information for each group. Patients with Tourette's syndrome were overall significantly slower (97 ms) in initiating movements than controls $(68 \mathrm{~ms})(F(1,22)=7 \cdot 44, \mathrm{p}<$ $0 \cdot 05)$. Despite this, there was no significant interaction between group and advance information $(F(2,44)=0.84, \mathrm{p}>0.44)$. Once again a trend analysis showed no evidence of progressive slowing over the three cue conditions for either group.

Thus patients with Tourette's syndrome were not dependent on external sensory cues to initiate movements. Despite this, patients with Tourette's syndrome were overall considerably slower $(97 \mathrm{~ms})$ in initiating movements than controls $(68 \mathrm{~ms})$, indicating that they were not preparing as effectively for the next response in the sequence.

\section{Movement time and sequencing effects}

Figure 4 also provides MT data. Patients with Tourette's syndrome were overall significantly slower $(330 \mathrm{~ms})$ in executing movements than controls $(262 \mathrm{~ms})(F(1,22)=10 \cdot 30, \mathrm{p}<$ $0 \cdot 01)$. There was also a significant main effect for advance information $(F(1,22)=540, \mathrm{p}<$ 0.001 ) and a highly significant group by advance information interaction $(F(2,44)=$ $7.89, \mathrm{p}<0.001)$. Post hoc one way ANOVAs and Tukey tests both established that patients with Tourette's syndrome and controls showed significant differences between each of the three levels of advance information. Patients with Tourette's syndrome showed significant $(\mathrm{p}<0.01)$ differences between low $(418 \mathrm{~ms})$ and moderate $(346 \mathrm{~ms})$, and between moderate and high (225 ms), and control subjects showed significant $(p<0.01)$ differences between low ( $329 \mathrm{~ms})$ and moderate $(281 \mathrm{~ms})$, and between moderate and high $(177 \mathrm{~ms})$ levels of advance information. It is clear, however, that the locus of the significant group by advance information interaction resides in the progressively smaller difference between subject groups, from low $(89 \mathrm{~ms})$ via moderate $(65 \mathrm{~ms})$ to high $(48 \mathrm{~ms})$ levels of advance information. Thus patients with Tourette's syndrome were most disadvantaged with low levels of advanced information. A trend analysis showed that there was no progressive slowing for either group, or for any condition.

The error rates for both groups increased slightly with high levels of advance information, compared with the other two conditions. The median errors for the three conditions (low, moderate, high) for patients with Tourette's syndrome were 0,1 , and 3, respectively, and for controls were 0,0 , and 2 respectively. Mann-Whitney $U$ tests showed that these group differences were not significant. 


\section{Medication}

Although there was a significant main effect of medication (medicated $v$ not medicated) $(F(1,10)=6.87, \mathrm{p}<0.05)$, the medicated group being $82 \mathrm{~ms}$ slower overall than the not medicated group, and a significant main effect of advance information $(F(1,10)=246.9, \mathrm{p}<$ $0.001)$, there was no significant interaction of medication by advance information $(F(2,20)$ $=2.57, \mathrm{p}>0.10)$. The magnitude of the major experimental effect of interest (the difference between the first and third conditions) was a little larger for the medicated group $(213 \mathrm{~ms})$ than for the not medicated group (174 ms).

\section{Depression}

There was no significant main effect of depression $(F(1,10)=0.03, \mathrm{p}>0.87)$; there was a significant main effect of advance information $(F(1,10)=212.65, \mathrm{p}<0.001)$. Most importantly, there was no significant interaction of depression by advance information $(F(2,20)=0.83, \mathrm{p}>0.45)$. The difference between the first and third condition was similar for the depressed $(202 \mathrm{~ms})$ and for the not depressed (185 ms) group. Thus irrespective of whether patients with Tourette's syndrome were depressed or not, they experienced most difficulties with low levels of advance information.

\section{Discussion}

This study employed two techniques, previously used for patients with Parkinson's disease $^{10}$ and Huntington's disease, ${ }^{12}$ to vary the amount of advance information in a sequential decision task, with a third group of patients with a disorder (Tourette's syndrome), which is also thought to affect the basal ganglia. ${ }^{6}$ The findings can be summarised as follows: with the first task (reliance on advance information) patients with Tourette's syndrome, like those with Parkinson's disease, were sensitive to reduced advance information in terms of MT, not DT; moreover, as predicted, under conditions of maximum reduction, responses of patients with Tourette's syndrome, again like those of patients with Parkinson's disease, progressively slowed with each successive element in the response sequence. With the second task (utilisation of advance information), again as predicted, patients with Tourette's syndrome, like patients with Parkinson's disease and Huntington's disease, were considerably disadvantaged at the level of response execution (MT), with low levels of advance information. Contrary to our prediction, however, and unlike the other two patient groups, their performance with high levels of advance information-that is, when both of the next two moves had to be simultaneously remembered during progress along the board-was no worse than when an intermediate level was provided. Sensory information might therefore become important to patients with Tourette's syndrome more at the execution phase of a movement sequence than at the ini- tiation phase. Thus although patients with Tourette's syndrome were able to initiate movements effectively over the three cue conditions, in the execution phase of the response cycle they were relatively more disadvantaged than controls with low levels of advance information.

Various explanations can be put forward to explain our findings. Firstly, attention deficit hyperactivity disorder is commonly manifested in Tourette's syndrome, and can often persist after tics have subsided in around $60 \%$ of cases. ${ }^{1}$ Patients with Tourette's syndrome may therefore require external visual cues to direct attention, and to control ongoing movements. This explanation is compatible with our findings, in that patients with Tourette's syndrome were considerably slower than controls in executing movements with high reductions in advance information, and with low levels of advance information. Conversely, with the other two conditions for each task (no and moderate levels of reduction, and moderate and high levels of advance information), MT correspondingly decreased, perhaps indicating that external sensory cues were assisting patients with Tourette's syndrome in directing their attention to given targets. In the present study, it is also noteworthy that patients with Tourette's syndrome exhibited evidence of attentional deficits in switching (trail making test B) and in maintaining attention (Stroop). The extent to which difficulty in sustaining and focusing attention might play a part in contributing to deficits on a variety of tasks in Tourette's syndrome requires further investigation. Indeed, we are currently considering this issue with our vibrotactile reaction time technique, which manipulates stimulus probability and validity of precueing. ${ }^{19}$

Secondly, without any reduction in advance information, prior programming and movement planning may be facilitated, as subjects can now rely on sensory guidance to initiate and execute a motor programme. With a high level of reduction in advance information, however, movements need to be planned and programmed in advance, especially when the target path disappears from view ahead of each next move. With limited visual guidance, patients with Tourette's syndrome may for some reason require more time to plan each next submovement, and consequently become less efficient in sequencing movements progressively down the board.

Finally, the basal ganglia are believed to provide an internal timing cue, via the ventral lateral thalamus, to the supplementary motor area for it to release (via the primary motor cortex) each stage in a response sequence..$^{20}$ In two such superficially dissimilar basal ganglia diseases as Parkinson's disease and Huntington's disease, this process may be affected, with the patient becoming more reliant on external cueing, ${ }^{12}{ }^{13}$ probably via the lateral premotor cortex. ${ }^{22}$ The present study suggests that patients with Tourette's syndrome may also be impaired in the sequenc- 
ing of elements in motor tasks that require the accessing of internal cues, and may be more or less unimpaired when external cues are provided. Visual stimuli can signal the arrival at each point in a sequence, thereby providing the necessary trigger cues for switching between movement sequences. When such stimuli extinguish in advance, internal cues generated by the basal ganglia are probably required for such switching. Basal ganglia dysfunction may impair the ability to use such internally generated cues to guide movements. Although the pathogenesis of Tourette's syndrome is still speculative, and alternative accounts have been proposed for the disorder, the present findings are compatible with the hypothesis of disorder of the basal ganglia in Tourette's syndrome.

Parkinson's disease causes a degeneration of dopaminergic neurons between the substantia nigra and the striatum, ${ }^{23}$ whereas Huntington's disease causes a progressive loss of small spiny projecting neurons in the caudate and putamen. ${ }^{24}$ Huntington's disease and Parkinson's disease share an underlying hypokinesia that may be masked in the early stages of the disorder by unwanted choreiform activity, the analogue of the tics of Tourette's syndrome. Tourette's syndrome is thought to involve a chemical imbalance in the lenticular nuclei (putamen and globus pallidus) and their interconnections with the frontal lobes. ${ }^{12}$ Although these three disorders may have their origin in common basal ganglia structures, specific sites and modes of impairment differentiate between these diseases. Kurlan ${ }^{25}$ suggests that Tourette's syndrome may be due to an abnormal gene causing inappropriate dopaminergic and serotonergic modulation, thereby leading to the defective operation or development of the basal ganglia and frontal and limbic systems. Disturbances of limbic activity and the modulation of the direct and indirect pathways through the basal ganglia may contribute to the abnormalities in Tourette's syndrome. ${ }^{125} \mathrm{We}$ believe the evidence is overwhelming that in Tourette's syndrome there is a dysfunction of the basal ganglia and their interconnections with the frontal lobes, although alternative hypotheses may still be entertained.

The question as to whether tics themselves may have affected Tourette's syndrome performance needs to be considered. It is well established that attentional and task related concentration tends to suppress the tics in Tourette's syndrome ${ }^{25}$ and, in any case, any movements that were disrupted by tics were excluded from the analysis. Indeed, it is difficult to see how tics could cause a systematic sequential effect in certain conditions of minimal advance information. We have also excluded a major influence from either medication or depression as contributing to the observed pattern of results. Irrespective of medication or depressive state, patients with Tourette's syndrome experienced a performance decrement when obliged to rely on limited levels of advance information. Moreover, in a recent study, ${ }^{26}$ comparing the movements of depressed patients with those of patients with movement disorders (Parkinson's disease) who were not depressed, it was reported that depressed patients showed no progressive slowing of movements, unlike the parkinsonian patients. Our present findings showed that patients with Tourette's syndrome, depressed or not, showed progressive slowing of movement, especially with the most reduced level of advance information.

In conclusion, basal ganglia dysfunction is thought to disrupt normal internal cueing mechanisms. There is compelling evidence in support of the proposition that Tourette's syndrome may result from abnormalities of the frontal basal-ganglionic circuit. These abnormalities may result in deficits in motor programme construction, and in programming and planning movement sequences in the absence of external visual cues, although our findings are compatible with alternative mechanisms for the disorder. Thus patients with Tourette's syndrome, regardless of whether medicated or depressed, may require more time to plan and programme motor strategies, and with limited visual guidance may require external visual cues to direct attention effectively to given targets.

This work was supported by grants from the Australian Brain Foundation and Australian Research Council. We gratefully acknowledge the help and cooperation of the Victorian Tourette Syndrome Association and all of its members who Wour Wood, Frank Devlin, Truong Nguyen, and Mike Durham for designing and maintruining the apparatus and software.

1 Channon S, Flynn D, Robertson MM. Attention deficits in Gilles de la Tourette syndrome. Neuropsychiatry, Neuropsychology, and Behavioural Neurology 1992;5: 170-7.

2 Commings DE. Tourette syndrome and human behavior, California: Hope Press, 1990.

3 Devinsky O, Geller BD. Gilles de la Tourette's syndrome. In: Joseph $\mathrm{AB}$, and Young RR, eds. Movement disorders In: Joseph $\mathrm{AB}$, and Young $\mathrm{RR}$, eds. Movement disorders in neurology and neuropsychiatry,

4 Devor EJ. Untying the Gordian knot: The genetics of Tourette syndrome. F Nerv Ment Dis 1990;178:669-79.

5 Shapiro AK, Shapiro ES, Young JG, Feinberg TE. Gilles de la Tourette syndrome. 2nd ed. New York: Raven Press, 1988 .

6 Singer HS, Walkup JT. Tourette syndrome and other tic disorders: diagnosis, pathophysiology, and treatment. Medicine 1991;70:15-32.

7 Hallett $M$. Physiology of basal ganglia disorders: An overview. Can ₹ Neurol Sci 1993;20:177-83.

Petersen B, Riddle MA, Cohen DJ, et al. Reduced basal ganglia volumes in Tourette's syndrome using threeganglia volumes in Tourette's syndrome using threedimensional reconstruction techniques from

9 Singer HS, Reiss AL, Brown JE, et al. Volumetric MRI Singer HS, Reiss AL, Brown JE, et al. Volumetric MRI changes in basal ganglia of childre
syndrome. Neurology 1993;43:950-6.

10 Georgiou N, Bradshaw JL, Iansek R, Phillips JG, Mattingley JB, Bradshaw JA. Reduction in external cues and movement sequencing in Parkinson's disease f Neurol Neurosurg Psychiatry 1994;57:368-70

11 Georgiou $\mathrm{N}$, Iansek $\mathrm{R}$, Bradshaw JL, Phillips JG Mattingley JB, Bradshaw JA. An evaluation of the role of internal cues in the pathogenesis of parkinsonian hypokinesia. Brain 1994;116:1575-87.

12 Bradshaw JL, Phillips JG, Dennis C, et al. Initiation and execution of movement sequences in those suffering from and at-risk of developing Huntington's disease. from and at-risk oxp Neuropsychol 1992;14:179-92.

13 Jones DL, Phillips JG, Bradshaw JL, Iansek R, Bradshaw JA. Programming of single movements in Parkinson's JA. Programming of single movements in Parkinson's Exp Neuropsychol 1992;14:762-72.

14 Ameican Psychiatic Association Diagnostic and Statistical Manual of Mental Disorders. 3rd ed. Washington, DC: Manual of Mental Disorders. 3rd

15 Kokmen E, Smith GE, Petersen RC, Tangalos E, Ivnik RC. The short test of mental status: correlations with RC. The short test of mental status: correlations with
standardised psychometric testing. Arch of Neurol 1991; 48:725-8. 
16 Yesavage JA, Brink TL, Rose TL, et al. Development and validation of a geriatric depression rating scale: a preliminary report. F Psychiatr Res 1983;17:37-49.

17 Reitan RM. Validity of the trail making test as an indication of organic brain damage. Percept Mot Skills 1958;8: 271-6.

18 Stroop JR. Studies of interference in serial verbal reactions. f Exp Psychol 1935;18:643-62.

19 Bradshaw JL, Waterfall ML, Phillips JG, Iansek R, Mattingley JB, Bradshaw JA. Reorientation of attention Mattingley JB, Bradshaw JA. Reorientation of attention
in Parkinson's disease: An extension to the vibrotactile in Parkinson's disease: An extension to the

20 Brotchie P, Iansek R, Horne MK. Motor function of the monkey globus pallidus: Neuronal discharge and parameters of movement. Brain 1991;114:1667-83.

21 Brotchie P, Iansek R, Horne MK. Motor function of the monkey globus pallidus: cognitive aspects of movement and phasic neuronal activity. Brain 1991;114:1685-1702.
22 Mushiake $H$, Inase $M$, Tanji J. Neuronal activity in the primary premotor supplementary and precentral motor cortex during visually guided and internally determined sequential movements. F Neurophysiol 1991;66:705-18.

23 Schell GR, Strick PL. The origin of thalamic inputs to the arcuate premotor and supplementary motor areas. $\mathcal{F}$ Neurosci 1984;4:539-60.

24 Shoulson I. Huntington's disease: cognitive and psychiatric features. Neuropsychiatry Neuropsychology and atric features. Neuropsychiatry Neur
Behavioural Neurology 1990;3:15-22.

25 Kurlan R. The pathogenesis of Tourette's syndrome: a possible role for hormonal and excitatory neurotransmitter influences in brain development. Arch Neurol 1992; 49:874-6.

26 Fleminger $S$. Control of simultaneous movements distinguishes depressive motor retardation from Parkinson's disease and neuroleptic parkinsonism. Brain 1992;115: 1459-80.

\section{Some speech disorders}

I have found three descriptions of dysphasia in the books of Dickens. Cleopatra Skewton is probably dysarthric, and perhaps there is a suggestion that her motor disability is bilateral, but she clearly makes dysphasic errors in speech and there is one suggestion that her limb disability has been right sided. Sir Leicester Dedlock suffers a hemiparesis (the side is not referred to), after a transient ischaemic attack. Both he and Cleopatra Skewton (a typical example of Dickens' sometimes grotesque humour) fare rather better when writing suggesting that Dickens, at least in the case of Sir Leicester, assumed that the written speech of dysphasic subjects would be free of errors. If Dickens was describing the occurrence of dysphasia in association with a right hemiparesis, he antedated Broca's description, though not that of Dax.

Sinclair Lewis inadvertently provides the sentence commonly used for assessing repetition in aphasic patients and Joyce and Wodehouse give examples of phrases commonly used, at least by earlier generations of neurologists, for the assessment of dysarthria. I have not found the original source for such phrases as West Register Street, Irish Constabulary, and British Constitution although all are mentioned in MonradKrohn. ${ }^{2}$

The unfortunate Mr Hodgkins, as described by Richard Gough's somewhat idiosyncratic spelling, would seem to have developed a permanent cerebellar dysarthria as the consequence of alcoholism, as perhaps may be the fate of Webster.

Richard Gough, 1834, The History of Myddle

This Mr Hodgkins made it his practice to goe to the alehouse dayley, and when hee came home drunke hee could goe as well as when hee was sober; but hee could not speake as others might understand him and att last hee had gott an habite of mashing att all times, soe that when hee was sober a man could hardly understand him.

Charles Dickens, 1843-4, Martin Chuzzlewit

He spoke to them in something of his own voice too, but sharpened and made hollow, like a dead man's face. What he would have said, God knows. He seemed to utter words, but they were such as man had never heard. And this was the most fearful circumstance of all, to see him standing there, gabbling in an unearthly tongue.

Charles Dickens, 1846-8, Dombey and Son Powerful remedies were resorted to; opinions given that she would rally from this shock, but would not survive another; and there she lay speechless, and star- ing at the ceiling, for days; sometimes making inarticulate sounds in answer to such questions as did she know who were present, and the like: sometimes giving no reply either by sign or gesture, or in her unwinking eyes.

At length she began to recover consciousness, and in some degree the power of motion, though not yet of speech. One day the use of her right hand returned; and showing it to her maid who was in attendance on her, and appearing very uneasy in her mind, she made signs for a pencil and some paper.... After much painful scrawling and erasing, and putting in of wrong characters, which seemed to tumble out of the pencil of their own accord, the old woman produced this document: "Rose-coloured curtains.". .

"Now, my dearest Grangeby," said Mrs Skewton, "You must posively prom," she cut some of her words short, and cut out others altogether, "come down very soon."... "Sterious wretch, who's he?" lisped Cleopatra.... "My dearest Edith-Grangeby-it's most trordinry thing," said Cleopatra, pettishly,.... "I won't have vistors-really don't want vistors," she said; "Little repose-and all that sort of thing - is what I quire. No odious brutes must proach me till I've shaken off this numbness;"...

Charles Dickens, 1853, Bleak House

Something frozen and fixed is upon his manner, over and above its usual shell of haughtiness, and $\mathrm{Mr}$ Bucket soon detects an unusual slowness in his speech, with now and then a curious trouble in beginning, which occasions him to utter inarticulate sounds.... But now he can only whisper, and what he whispers sounds like what it is-mere jumble and jargon.... After vainly trying to make himself understood in speech, he makes signs for a pencil. So inexpressively that they cannot at first understand him.... The slate comes into requisition again, but the word he wants to write he cannot remember. ... .

Sir Leicester, lying in his bed, can speak a little, though with difficulty and indistinctness.

"No. In addition to my older malady, I have had a sudden and bad attack. Something that deadens," making an endeavour to pass one hand down one side, "and confuses," touching his lips. . .

Arnold Bennett, 1907, The ghost

At the age of three, just as I was beginning to talk easily, I became, for a period, subject to fits; and in one of these I lost the power of speech. ... I could hear and understand, but I could not speak. (He regains his speech when he finds his father's hanging body.) 